\title{
The effects of intermittent hypoxia on human nasal mucosa
}

\author{
Seung Min $\mathrm{In}^{1} \cdot$ Do-Yang Park ${ }^{2} \cdot \mathrm{Ki}-\mathrm{II} \mathrm{Lee}^{1} \cdot$ Gayoung Gu${ }^{2} \cdot$ Hyun Jun $\mathrm{Kim}^{2}$ (D)
}

Received: 15 June 2020 /Revised: 15 December 2020 / Accepted: 18 December 2020 / Published online: 2 January 2021

(C) The Author(s), under exclusive licence to Springer Nature Switzerland AG part of Springer Nature 2021

\begin{abstract}
Purpose Intermittent hypoxia (IH) is characterized by hypoxia-reoxygenation, reported to be a critical risk factor for obstructive sleep apnea (OSA). This experiment aimed to evaluate the direct effects of IH on the human nasal mucosa.

Methods The direct effects of IH on the human nasal mucosa was evaluated by measuring the ciliary beat frequency (CBF) and expression levels of inflammatory cytokines (granulocyte-macrophage colony-stimulating factor, transforming growth factor- $\beta$, interleukin-6, and tumor necrosis factor- $\alpha$ ). The normoxia group was exposed to a normoxic condition for $72 \mathrm{~h}$. The IH group was exposed to 288 cycles of IH ( 1 cycle: hypoxia, $5 \mathrm{~min}$; subsequent normoxia, $10 \mathrm{~min}$ ) for $72 \mathrm{~h}$. CBF was measured using an automated computer-based video image processing technique. Changes in the expression of cytokines were assessed by real-time reverse transcription-polymerase chain reaction (RT-PCR).

Results The normoxia group revealed a persistent CBF pattern and a physiological range of inflammatory cytokines. However, the IH group showed a cyclic decrease in CBF and increased expression of inflammatory cytokines. Cytotoxicity assay indicated no difference in the survival rates between the two groups.

Conclusions IH results in increased expression of inflammatory cytokines that adversely affects the mucociliary transport in the upper airway and, consequently, may result in airway inflammation.
\end{abstract}

Keywords Obstructive sleep apnea $\cdot$ Hypoxia/reoxygenation $\cdot$ Inflammation $\cdot$ Ciliary beat frequency $\cdot$ Cytokine

\section{Introduction}

Obstructive sleep apnea (OSA) is a highly prevalent lifelong disorder, which is characterized by repetitive upper airway obstruction during sleep and recurrent episodes of oxygen desaturation. The pathological mechanism of OSA has mainly focused on intermittent hypoxia (IH), which oscillates through periods of hypoxia and reoxygenation. IH is a condition in which the human body is temporarily deprived of adequate blood oxygen supply. During episodes of airway obstruction, the oxygen levels in the arterial blood decreases; the resultant hypoxia alleviates when the airway reopens. These repetitive cycles of hypoxia and subsequent reoxygenation may initiate

Hyun Jun Kim

entkhj@naver.com

1 Department of Otorhinolaryngology-Head and Neck Surgery, Konyang University College of Medicine, Daejeon, Republic of Korea

2 Department of Otolaryngology, Ajou University School of Medicine, Suwon, Republic of Korea a pathological cascade characterized by sympathetic activation, oxidative stress, endothelial dysfunction, and metabolic disorders [1-3]. However, no studies have examined the direct role of $\mathrm{IH}$ on the human respiratory mucosa.

The pathogenesis of OSA is a multifactorial process, involving a variety of mechanisms, including the selective activation of the upper airway inflammatory pathways. Reports indicate that patients with OSA develop upper airway inflammation [4-6]. Cytokines play an essential role in the pathophysiology of respiratory mucosal inflammations [7]. In patients with OSA, several inflammatory markers are present in high concentrations, such as interleukin-6 (IL-6), IL-8, vascular endothelial growth factor (VEGF), tumor necrosis factor- $\alpha$ (TNF- $\alpha$ ), and C-reactive protein (CRP) [8]. Furthermore, IL6 , TNF- $\alpha$, and CRP levels have been shown to decrease after treatment of OSA with surgical intervention and/or continuous positive airway pressure [9]. These findings suggest that OSA may induce airway inflammation through the activation of various pro-inflammatory cytokines.

Mucociliary clearance refers to a process in which coordinated waves of ciliary beats move a blanket of mucus progressively upward along the airway mucosa. Many chronic 
medical conditions, such as OSA, may have detrimental effects on the mucociliary transport. In particular, upper airway obstruction has been associated with decreased mucociliary clearance rate [10]. However, few studies have investigated the association between OSA and mucociliary dysfunction $[11,12]$. To provide additional evidence in support of upper airway inflammation, we measured ciliary beat frequency (CBF) in the setting of chronic IH of OSA.

The authors hypothesized that increased expression of inflammatory cytokines and decreased CBF may be key mechanisms linking OSA and upper airway inflammation. To assess the direct effects of IH on upper airway inflammation, $\mathrm{CBF}$ and expression of inflammatory cytokines were evaluated in the experimental setting of chronic IH. The primary purpose of the study was to evaluate the direct effects of $\mathrm{IH}$ on the upper airway mucosa by measuring the CBF and the expression of inflammatory cytokines in experimental chronic IH.

\section{Methods}

\section{Study subjects}

A total of healthy 18 adults, 19-70 years of age, were assessed for participation in this study. This study was a single institutional study and approved by the Human Research Ethics Committee of the Ajou University Hospital. The protocol was approved by the Ajou IRB (IRB no: AJIRB-MEDSMP-12-223) and was conducted in accordance with the 1964 Helsinki Declaration. Informed written consent was obtained from all subjects prior to their inclusion in the study. We included adult patients who underwent sinonasal surgery for rhinologic symptom, sinonasal biopsy, or rhinoseptoplasty in our department and excluded smokers, patients with asthma, on any medication, and respiratory illness, including recent episodes of cold.

\section{Normal human nasal mucosa culture}

Nasal mucosa of 18 patients was obtained. The mucosal samples were washed and cut into $0.5 \times 0.5$ sized pieces. The samples were then cultured in 6-well cell culture plates and stabilized in an incubator at $5 \% \mathrm{CO}_{2}, 100 \%$ humidity, and 37 ${ }^{\circ} \mathrm{C}$ for 24 hours. The cultured nasal mucosa samples were exposed to normoxia and IH conditions. These resultant cell cultures were used for histological examination and to measure the $\mathrm{CBF}$ after exposure to $\mathrm{IH}$.

\section{Normal human nasal epithelial cell culture}

Inferior turbinate samples were collected using the procedures for collecting nasal mucosa as previously described. Passage-
2 NHNE cells $\left(6 \times 10^{5}\right.$ cells/well $)$ obtained as described above were seeded in a $0.5 \mathrm{~mL}$ of culture medium on $24.5-\mathrm{mm}$ diameter, $0.45-\mu \mathrm{m}$ pore size Transwell-Clear culture inserts. The NHNE cells were cultured and submerged for 1 week. The culture medium was changed daily subsequent to the development of the air-liquid interface, and the experiments on NHNE cells were performed after 14 days. The cultures were stabilized $\mathrm{c}$ in an incubator at $37^{\circ} \mathrm{C}$, with a humidified $5 \% \mathrm{CO}_{2}$ atmosphere for 1 day. The NHNE cell cultures were exposed to conditions emulating normoxia and $\mathrm{IH}$. These normoxia- and IH-exposed cultures were used for both the cytokine study and MTT (3-(4,5-dimenthylthiazol-2yl)-2,5diphenyl-2H-tetrazolium bromide) assay.

\section{MTT assay}

The MTT assay was performed to evaluate the metabolic activity of viable cells in IH-exposed NHNE cells. The cultured cells were seeded into 96 well plates, incubated at $37^{\circ} \mathrm{C}$ in $5 \%$ $\mathrm{CO}_{2}$ atmosphere, and then challenged to IH conditions. MTT solution (Sigma, St. Louis, MI, USA) was mixed to the culture medium, and the samples were fostered in a same setting for $48 \mathrm{~h}$. Then, the absorbance of the treated cells was measured at $540 \mathrm{~nm}$

\section{Exposure to hypoxic condition}

Our intermittent hypoxia system is a custom-designed computer-controlled incubation system. The authors used a specially designed exposure chamber to generate hypoxic condition and to provide a constant temperature, humidity, and airflow. The chamber is automatically supplied with mixed gases (oxygen, nitrogen, and carbon dioxide) according to the desired condition. This system enables a variety of studies on OSA under anoxia, hypoxia, and normoxia (Fig. 1). In the normoxia group, $21 \% \mathrm{O}_{2}$ (atmospheric oxygen concentrations, $21 \% \mathrm{O}_{2} ; 5 \% \mathrm{CO}_{2}, 37^{\circ} \mathrm{C}$ ) was supplied continuously for 3 days. In the IH group, the cultured nasal mucosal cells were exposed to both normoxia $(16 \mathrm{~h})$ and $\mathrm{IH}(8 \mathrm{~h})$ conditions for 3 days, similar to that experienced by a patient with OSA. The IH condition was simulated by subjecting each IH chamber with programmed, alternating normoxic (10 min, 21\% $\mathrm{O}_{2}$ ), and subsequent hypoxic $\left(5 \mathrm{~min}, 5 \% \mathrm{O}_{2}\right)$ conditions. Each IH cycle lasted $15 \mathrm{~min}$ : first $10 \mathrm{~min}$ of normoxia followed by the next $5 \mathrm{~min}$ of hypoxia. These IH cycles were repeated for the whole duration of the IH period ( $8 \mathrm{~h} / 1$ day) for 3 days (Fig. 2).

\section{CBF response after exposure to IH}

CBF measurement was performed as delineated previously [13]. In short, a peak detection method for CBF measurement and an optical flow technique-based program were used [14]. 


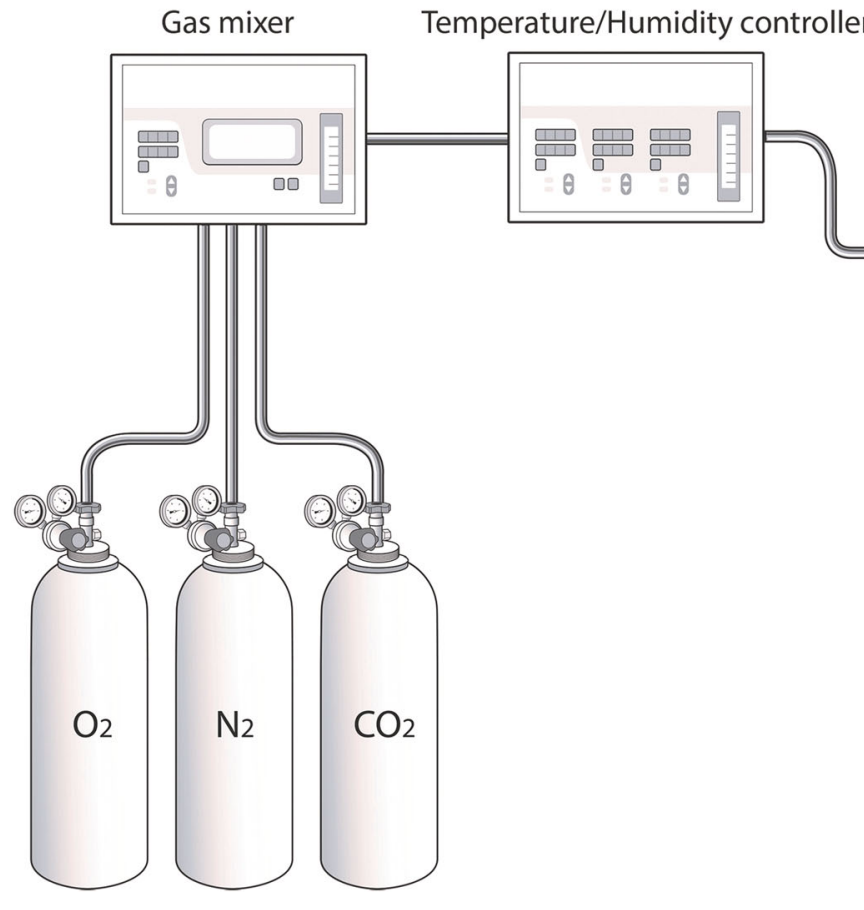

Fig. 1 Experimental diagram. The intermittent hypoxia (IH) system is composed of a custom-designed, computer-controlled incubating chamber and gas mixer $\left(\mathrm{O}_{2}, \mathrm{~N}_{2}\right.$, and $\left.\mathrm{CO}_{2}\right)$. Cultured cells were exposed to $\mathrm{IH}$

Then, ciliated cells were magnified with a $\times 400$ objective under an inverted microscope. The ciliary beating was captured with a digital camera and projected on a monitor. The developed program automatically measured the $\mathrm{CBF}$ from the or normoxia, and the ciliary movements were measured by using an optical flow technique with a peak detection method under an inverted microscope

recorded data. $\mathrm{CBF}$ was assessed in at least 5-6 different sites, and the mean value was considered a patient's CBF. CBF data was analyzed using manual counts as well as the program. No differences were observed between the two methods of
Fig. 2 Exposure to hypoxia. In the normoxia group, continuous $21 \% \mathrm{O}_{2}$ was supplied for 3 days. In the IH group, the nasal mucosa cultured cells were exposed to both normoxia $(16 \mathrm{~h})$ and $\mathrm{IH}(8 \mathrm{~h})$ for 3 days. Each cycle of IH lasted $15 \mathrm{~min}$ : first $10 \mathrm{~min}$ of normoxia and followed by 5 min of hypoxia. These cycles of IH were repeated for the whole duration of IH exposure $(8 \mathrm{~h})$ for 3 days

\section{Normoxia (N)}

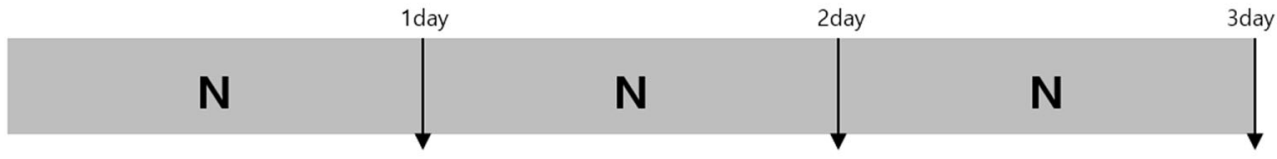

\section{Intermittent Hypoxia (IH)}

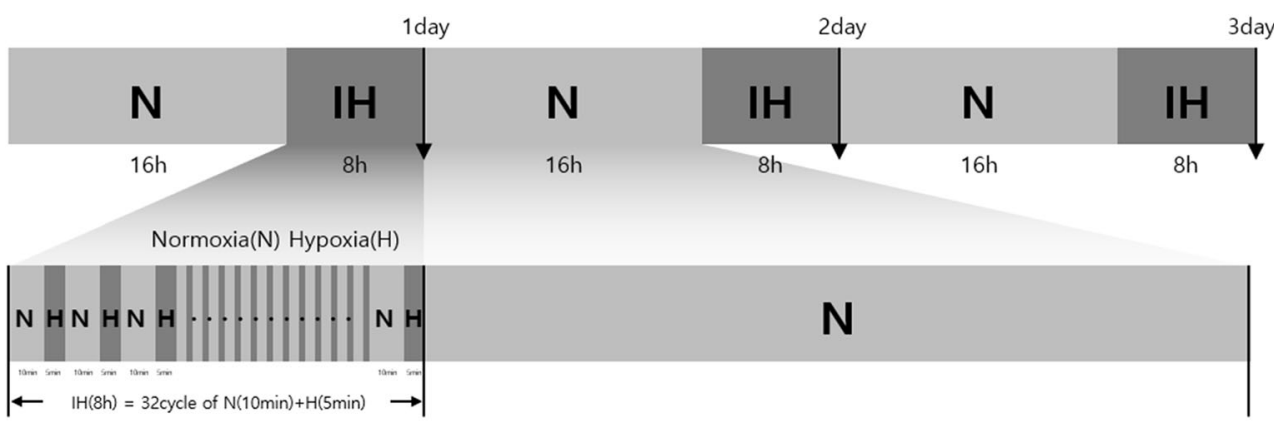


analysis. The human nasal mucosal cell cultures were exposed to normoxic and IH conditions, and CBF was measured for 3 days.

\section{IH-induced changes in the expression of inflammatory cytokines assessed by RT-PCR}

The mRNA expression (GM-CSF, TGF- $\beta$, IL-6, and TNF- $\alpha$ ) of NHNE cells exposed to IH was confirmed by RT-PCR. Total RNA was extracted from NHNE cells using the RNA protect cell mini kit (Qiagen, Hilden, Germany) and FastPure RNA kit (Takara Bio, Otsu, Japan). cDNA was synthesized using total RNA as the template with a High Capacity cDNA Reverse Transcription Kit (Applied Biosystems, Foster City, CA, USA). Real-time PCR was performed using Fast SYBR ${ }^{\circledR}$ Green Master Mix (Applied Biosystems). The relative levels of specific cytokine mRNAs were calculated by real-time PCR. Reverse transcription was achieved by using $0.5 \mu \mathrm{g}$ of total RNA with Oligo(dT) ${ }_{23}$ and M-MLV Reverse Transcriptase (Life Technologies). Results for the specific mRNA expression were always presented as relative expression of the target mRNA with respect to that of the housekeeping.

\section{Histopathological examination}

To examine the morphological changes in IH-exposed upper airway nasal mucosa, $30 \mathrm{~min}$ and $2 \mathrm{~h}$ after hypochlorous acid $(\mathrm{HOCl})$ treatment, the cultured cells were embedded in $10 \%$ neutral-buffered formalin, fixed in paraffin, and sliced for histological examination. The sections were stained with hematoxylin-eosin and examined daily with a phase-contrast light microscope (Olympus Light Microscope, Vanox-S type, Japan).

\section{Statistical analyses}

Values are expressed as the mean \pm standard error of mean. The CBF response after IH exposure was evaluated using the Mann-Whitney $U$ test. Data of the MTT assay and RT-PCR about inflammatory cytokine expression were assayed using the student $t$-test. $P$ values $<0.05$ were considered statistically significant.

\section{Results}

\section{Demographics}

Overall, 18 adult subjects were included in this analysis. There was a similar number and percentage of male $(n=10 ; 55.6 \%)$ and female $(n=8 ; 44.4 \%)$ patients. The mean age of male and female were 41.9 and 43.4 years, respectively. The mean age of overall patients was 42.6 years.

\section{MTT assay}

Results of MTT assay showed no statistical significance in the viabilities of IH-exposed and non-exposed groups of cultured mucosal cells. This implies that IH exposure does not induce cytotoxicity (Fig. 3).

\section{CBF response after IH exposure}

Baseline CBF values of the normoxia IH groups were $16.75 \mathrm{~Hz}$ and $16.31 \mathrm{~Hz}$, respectively. CBF of the IHexposed group was less than that of the normoxia group. The CBF values did not change in the normoxia group. CBF of IH group began to reduce from the initial challenge of IH, with a maximal difference of $17 \%$. In the IH group, the decrease in $\mathrm{CBF}$ of each hypoxic group recovered to within the baseline $\mathrm{CBF}$ levels of the normoxia group during subsequent normoxia cycle. These repetitive, cyclic changes of CBF during hypoxia demonstrated a fluctuating pattern. This fluctuation continued throughout the whole IH exposure experiment during the 3-day period (Fig. 4).

\section{IH-induced changes of inflammatory cytokines by RT- PCR}

Exposure to IH resulted in a significant increase in the level of IL-6, TGF- $\beta$, GM-CSF, and TNF- $\alpha$ mRNAs $(p<0.05)$. Especially, IL-6 and GM-CSF mRNA levels showed more than a sevenfold increase (Fig. 5).

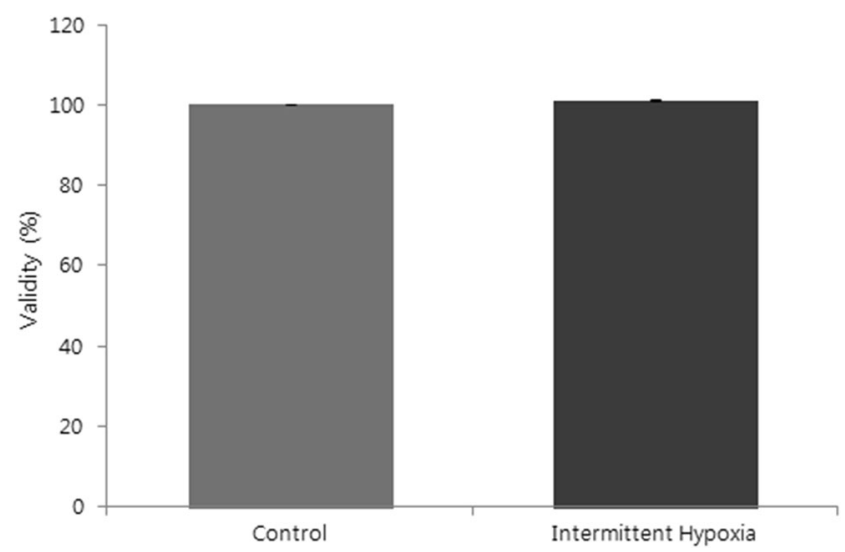

Fig. 3 MTT cell viability assay. NHNE cell cultures were exposed to IH, and the spectrophotometric absorbance was analyzed at $540 \mathrm{~nm}$. The control group was not challenged to $\mathrm{IH}$, although all the other conditions were identical. All data are expressed as the mean \pm standard error of mean. There was no significant difference between the control and $\mathrm{IH}$-exposed groups 


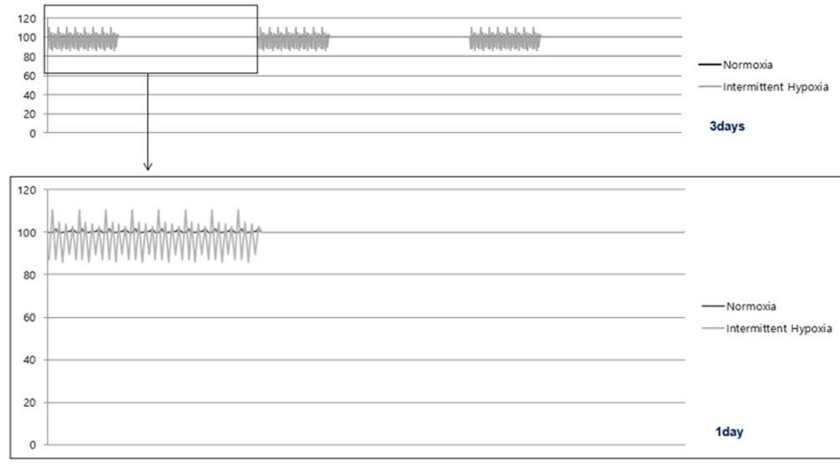

Fig. $4 \mathrm{CBF}$ response after IH exposure. In the normoxia group, there was no change in $\mathrm{CBF}$ values. $\mathrm{CBF}$ of $\mathrm{IH}$ group began to decrease from the initiation of first hypoxic exposure, with a maximal difference of $17 \%$. In the IH exposure group, the decrease in CBF with each hypoxic cycle

\section{Histopathological examination}

Histological examination of IH-exposed human nasal mucosal cultures revealed no differences as compared to that in the normoxia culture group (Fig. 6). This indicates that the IHexposed group was free of cellular tissue damage.

\section{Discussion}

OSA is characterized by reiterated events of upper airway obstruction during sleep that results in $\mathrm{IH}$, increased inspiratory efforts, and sleep fragmentation. IH is a pathological hallmark of OSA and is considered an important trigger for the

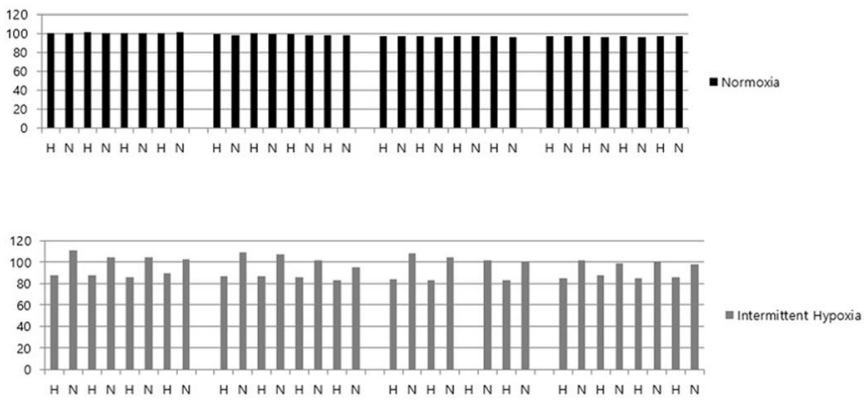

recovered nearly to that of the baseline $\mathrm{CBF}$ values of the normoxia group during the next normoxic cycle. These repetitive, cyclic changes in $\mathrm{CBF}$ values due to hypoxic exposure show a fluctuating pattern

manifestation of adverse effects in patients with OSA. Other pathophysiological changes in OSA include intermittent hypercapnia, oxidative stress, change in intrathoracic pressure, sympathetic activation, selective activation of airway inflammation, and sleep fragmentation $[8,15]$. Because of the synchronicity, it is not easy to distinguish the net effect of the two main manifestations - IH and sleep fragmentation - in clinical and laboratory studies of OSA. Therefore, the authors used $\mathrm{IH}$ as the only factor in this study.

The usual upper airway manifestations in patients with OSA include the oral discomfort, such as sore throat, dysphagia, and dry mouth. Although breathing during normal sleep is usually nasal rather than oral, patients with OSA often breathe through their mouth, and this may cause dryness of the mouth
Fig. 5 Measurement of inflammatory cytokines by real time RT-PCR. IH results in an significant increase in IL-6, TGF$\beta$, GM-CSF, and TNF- $\alpha$ mRNAs $(p<0.05)$. Especially, IL-6 and GM-CSF mRNA levels are elevated more sevenfold. All values are expressed as the mean \pm standard error of mean. $* p<0.05$, compared with normoxia group
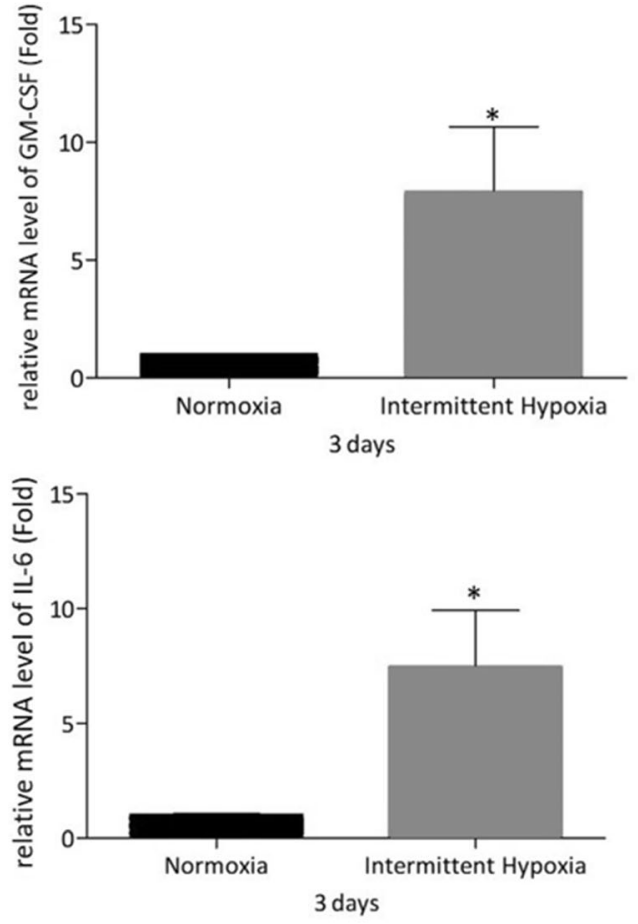
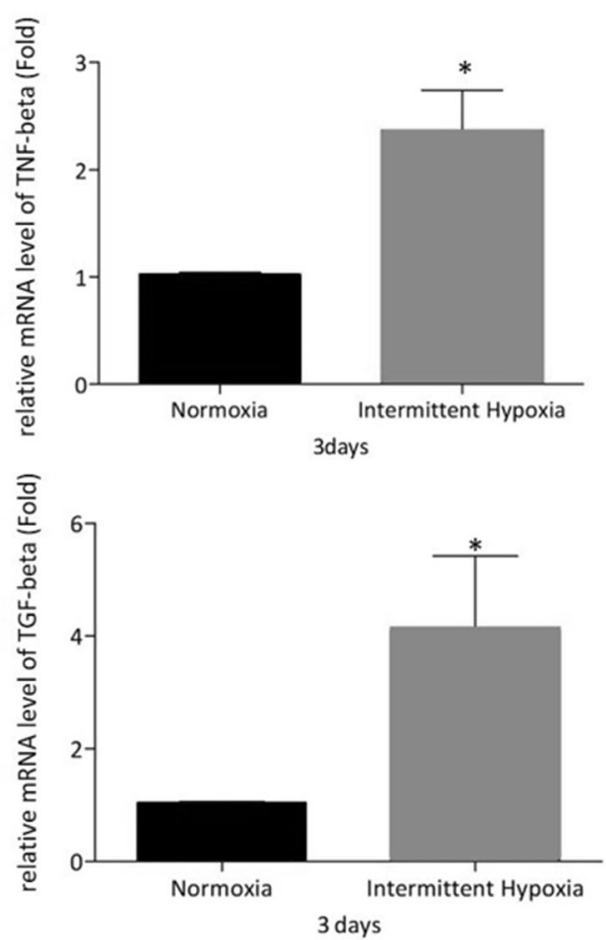
Fig. 6 Histopathological examination. Histological examination of the IH-exposed cells reveals no differences as compared to that in the normoxia culture group. a Normoxia $(x$ 200). b Normoxia $(\times 400)$. c IH $(\times$ 200). d IH $(\times 400)$

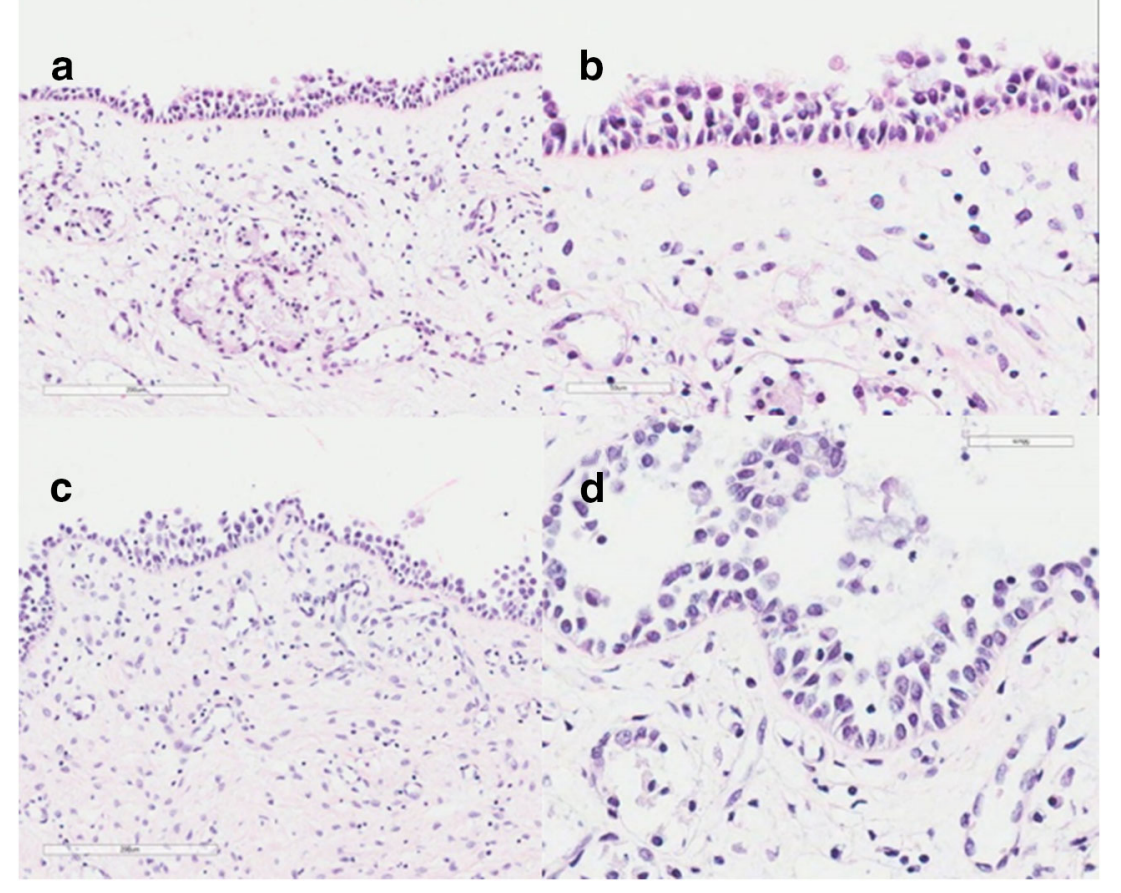

and throat. Directly, the oral breathing of dry air by patients with OSA leads to the excessive water loss by the nasal mucosa, which may subsequently attenuate the nasal mucociliary clearance [16]. If the mucociliary clearance is injured, the excess mucus leads to plugs and plaques in the upper respiratory airway. This condition in patients with OSA can have negative effects on the defense mechanisms of the upper airway and make them susceptible to airway inflammation. Moreover, patients with OSA present with upper airway obstruction symptoms characterized by pharyngeal airway collapse and repetitive events of upper airway blockage, peripheral deoxygenation, and awakening. These unfavorable conditions in OSA may negatively affect the mucociliary transport. However, only a few studies have investigated the association between OSA and mucociliary dysfunction $[11,12]$. Previous articles indicate that obstruction of the upper airway is associated with a decreased mucociliary clearance rate $[15$, 17]. Deinz et al., using the saccharine test in patients with severe OSA, showed that nasal mucociliary system is significantly deteriorated [10]. In contrast, Bossi et al. concluded that the CPAP usage in patients with severe OSA did not change the nasal function and mucociliary clearance of nasal epithelium in the experimental study [11]. Saka et al. also concluded that the histopathologic inflammation and fibrosis were increased after CPAP treatment in patients with OSA. In present study, CBF of the normoxia group was maintained constantly for 3 days. However, the hypoxic period in the IH group revealed approximately $17 \%$ reduction of $\mathrm{CBF}$. In another study, a fourfold correlation between CBF (16\%) and mucociliary transport (56\%) was shown [18]. Although mucociliary transport may not be directly proportional for $\mathrm{CBF}$, the fact that the change of $\mathrm{CBF}$ is smaller than that of mucociliary clearance remains common. In addition, our experiment only assessed the 3-day outcome, but the effect of actual lifetime accumulation of IH cannot be ignored. Therefore, mucociliary transport of the IH group might have been inhibited much more than $17 \%$.

Our work has focused on the hypothesis that chronic IH condition negatively affects mucociliary transport of the human nasal mucosa and may cause airway inflammation, thereby creating a vicious cycle that increases the tendency to develop serious upper airway infection, particularly sinonasal infection. This hypothesis was based on the fact that impaired CBF may result in many different airway diseases, such as common cold, bronchitis, and rhinosinusitis. The mucociliary transport system could be critical for defense mechanism against respiratory viral infections. In the experimental study from healthy subjects, Chilvers et al. reported that human coronavirus caused the damage of the ciliated epithelium [19]. The reduced $\mathrm{CBF}$ due to OSA can attenuate the mucociliary clearance against infectious substances such as virus and bacteria. This can result in increased susceptibility to respiratory diseases. During the current pandemic of novel coronavirus disease-2019 (COVID-19), patients with OSA must be especially careful for various viral infections including COVID-19. Also, chronic rhinosinusitis (CRS) is a common sinonasal disorder, and OSA has been reported to be a common manifestation in patients with CRS. Recently, Jiang RS et al. reported that patients with 
CRS have a high prevalence of OSA [20]. It is believed that nasal obstruction causes increased airway resistance resulting in the development of OSA. Moreover, it has been suggested that CRS is associated with the release of inflammatory cytokines, which could be involved in the development of OSA [21]. IH, the hallmark feature of OSA, results in preferential activation of inflammatory pathways that may contribute to inflammatory response of the upper airway. Prior studies propose that oxidative stress by IH induces the release of inflammatory cytokines [22-26].

OSA-associated IH is linked to systemic inflammation. The underlying mechanisms responsible for OSA are inconspicuous; however, the activation of systemic inflammation might be an essential role. Inflammatory cytokines are a broad category of small proteins that are important in cell signaling and airway inflammatory responses. Various studies have demonstrated that OSA is highly associated with inflammatory cytokines, including TNF- $\alpha$, IL-6, IL8, GM-CSF, TGF- $\beta$, $\beta$-actin, and CRP [22-26]. Various studies have reported that chronic hypoxia causes various inflammatory responses. However, it remains unclear whether IH directly affects the upper airway inflammation. Mechanical stress exerted on the respiratory tract mucosa by snoring and apnea may be responsible for airway inflammation. During sleep, patients with OSA experience repetitive deoxygenation-reoxygenation in peripheral blood. Immunologically, it is assumed that the exposure to IH during OSA increases oxidative stress and may invoke airway inflammations. However, further studies are needed to clarify the exact underlying mechanisms.

MTT assay was used to identify the cell death by IH. The results revealed no significant difference between two experimental groups. This suggests that IH had no cytotoxic effect and CBF reduction in the IH-exposed group was associated with decreased ciliary function. In our histopathological examination, IH-exposed human nasal mucosal cultures showed no differences as compared to that in the normoxia culture group. This indicates that the IHexposed group was free of cellular tissue damage.

There is some methodological limitation of the present study. Our IH cycle may not fully replicate the apnea condition experienced by patients with OSA. Ng et al. used the oxygen cycle with $21 \%$ and $5 \%$ every other alternate for $8 \mathrm{~h}$ per day for 7 days [27]. They applied more frequent and severe hypoxic condition of IH cycles which might cause the results to be exaggerated. Shiota et al. applied an oxygen cycle ( 8 cycles of $1 \% \mathrm{O}_{2}$ and $21 \% \mathrm{O}_{2}$ every $10 \mathrm{~min}$ ) for $8 \mathrm{~h}$ per day for 4 weeks in an in vitro study [28]. The experimental setting used in this study was particularly suitable for mimicking $\mathrm{IH}$ conditions in patients with OSA. We applied a more controlled pattern of 4 events (hypoxia and normoxia)/h than previous studies, with a shorter duration of hypoxia $(5 \mathrm{~min})$ than normoxia (10 min). However, we did not assess the specific effect of different time pattern of IH on the consequences of OSA.

The authors presume that the pressure difference caused by intermittent upper airway obstruction likely affects to the lower respiratory system as well. OSArelated mechanical episode may not be the sole mechanism underlying airway inflammation. In our study, the direct airway effect of IH was a cyclic decrease in CBF. Based on this result, mucociliary dysfunction by impaired CBF may lead to various respiratory tract disorders, and IH is more likely to cause upper airway inflammation in patients with OSA. Although our experiment only used 3 days of exposure to $\mathrm{IH}$, patients with OSA are presumed to be exposed to IH throughout their life, for several decades. Therefore, the adverse effects of chronic IH exposure would be increased over time. Thus, cumulative IH exposure might adversely affect patients with severe OSA.

Our study is solely based on in vitro experimentation. Therefore, the results may vary from that obtained from actual IH exposure of patients with OSA due to several factors. Future studies with in vivo experimental models and clinical human trials might demonstrate the adverse effects of $\mathrm{IH}$ in patients with OSA.

\section{Conclusion}

IH increased inflammatory cytokines and adversely affected the upper airway mucociliary transport. Clinically, these findings suggest that local IH can influence inflammatory diseases such as rhinitis, chronic sinusitis, or even viral infections in the upper airway.

Acknowledgments The authors of this paper hereby declare that Kim HJ received research funding from the Ministry of Science, ICT \& Future planning of Korea (Contract grant number: 2012-012474) and National Research Foundation of Korea Grant, funded by the Korean Government (NRF-2017R1E1A1A01074543).

Authors' contributions All authors have seen and approved the manuscript.

\section{Compliance with ethical standards}

Conflict of interest The authors declare that they have no conflict of interest.

Ethical approval All procedures performed in studies involving human participants were in accordance with the ethical standards of the institutional and/or national research committee and with the 1964 Helsinki Declaration and its later amendments or comparable ethical standards (IRB no: AJIRB-MED-SMP-12-223). 


\section{References}

1. Minguchi K, Tazaki T, Yokoe T, Minoguchi H, Watanabe Y, Yamamoto M, Adachi M (2004) Elevated production of tumor necrosis factor-alpha by monocytes in patients with obstructive sleep apnea syndrome. Chest 126(5):1473-1479. https://doi.org/ 10.1378/chest.126.5.1473

2. Svatikova A, Wolk R, Gami AS, Pohanka M, Somers VK (2005) Interactions between obstructive sleep apnea and the metabolic syndrome. Curr Diabetes Rep 5(1):53-58. https://doi.org/10.1007/ s11892-005-0068-2

3. Punjabi NM, Shahar E, Redline S, Gottlieb DJ, Givelber R, Resnick HE, Sleep Heart Health Study I (2004) Sleep-disordered breathing, glucose intolerance, and insulin resistance: the Sleep Heart Health Study. Am J Epidemiol 160(6):521-530. https://doi.org/10.1093/ aje/kwh261

4. Salerno FG, Carpagnano E, Guido P, Bonsignore MR, Roberti A, Aliani M, Vignola AM, Spanevello A (2004) Airway inflammation in patients affected by obstructive sleep apnea syndrome. Respir Med 98(1):25-28. https://doi.org/10.1016/j.rmed.2003.08.003

5. Rubinstein I (1995) Nasal inflammation in patients with obstructive sleep apnea. Laryngoscope 105(2):175-177. https://doi.org/10. 1288/00005537-199502000-00012

6. Sekosan M, Zakkar M, Wenig BL, Olopade CO, Rubinstein I (1996) Inflammation in the uvula mucosa of patients with obstructive sleep apnea. Laryngoscope 106(8):1018-1020. https://doi.org/ 10.1097/00005537-199608000-00021

7. Gosepath J, Schaefer D, Brommer C, Klimek L, Amedee RG, Mann WJ (2000) Subacute effects of ozone exposure on cultivated human respiratory mucosa. Am J Rhinol 14(6):411-418. https:// doi.org/10.2500/105065800779954275

8. de Lima FF, Mazzotti DR, Tufik S, Bittencourt L (2016) The role inflammatory response genes in obstructive sleep apnea syndrome: a review. Sleep Breath 20(1):331-338. https://doi.org/10.1007/ s11325-015-1226-7

9. Tam CS, Wong M, McBain R, Bailey S, Waters KA (2006) Inflammatory measures in children with obstructive sleep apnoea. J Paediatr Child Health 42(5):277-282. https://doi.org/10.1111/j. 1440-1754.2006.00854.x

10. Deniz M, Gultekin E, Ciftci Z, Alp R, Ozdemir DN, Isik A, Demirel OB (2014) Nasal mucociliary clearance in obstructive sleep apnea syndrome patients. Am J Rhinol Allergy 28(5):178-180. https:// doi.org/10.2500/ajra.2014.28.4094

11. Bossi R, Piatti G, Roma E, Ambrosetti U (2004) Effects of longterm nasal continuous positive airway pressure therapy on morphology, function, and mucociliary clearance of nasal epithelium in patients with obstructive sleep apnea syndrome. Laryngoscope 114(8):1431-1434. https://doi.org/10.1097/00005537-20040800000022

12. Sazak Kundi FC, Murat Ozcan K, Okudan B, Coskun N, Inan MA, Ozcan M (2020) Effects of chronic sleep deprivation on upper respiratory tract mucosal histology and mucociliary clearance on rats. J Sleep Res:e13065. https://doi.org/10.1111/jsr.13065

13. In SM, Kim HJ, Park RW, Kim W, Gimm YM, Park I, Hong S, Hong JJ, Oh JH, Kahng H, Park EY (2013) The effects of a $1.8 \mathrm{GHz}$ continuous electromagnetic fields on mucociliary transport of human nasal mucosa. Laryngoscope 123(2):315-320. https://doi.org/ 10.1002/lary.23620

14. Kim W, Han TH, Kim HJ, Park MY, Kim KS, Park RW (2011) An automated measurement of ciliary beating frequency using a combined optical flow and peak detection. Healthc Inf Res 17(2): 111-119. https://doi.org/10.4258/hir.2011.17.2.111

15. Uslu H, Uslu C, Varoglu E, Demirci M, Seven B (2004) Effects of septoplasty and septal deviation on nasal mucociliary clearance. Int J Clin Pract 58(12):1108-1111. https://doi.org/10.1111/j.17421241.2004.00205.x

16. Salah B, Dinh Xuan AT, Fouilladieu JL, Lockhart A, Regnard J (1988) Nasal mucociliary transport in healthy subjects is slower when breathing dry air. Eur Respir J 1(9):852-855

17. Gudis D, Zhao KQ, Cohen NA (2012) Acquired cilia dysfunction in chronic rhinosinusitis. Am J Rhinol Allergy 26(1):1-6. https:// doi.org/10.2500/ajra.2012.26.3716

18. Seybold ZV, Mariassy AT, Stroh D, Kim CS, Gazeroglu H, Wanner A (1990) Mucociliary interaction in vitro: effects of physiological and inflammatory stimuli. J Appl Physiol 68(4):14211426. https://doi.org/10.1152/jappl.1990.68.4.1421

19. Chilvers MA, McKean M, Rutman A, Myint BS, Silverman M, O'Callaghan C (2001) The effects of coronavirus on human nasal ciliated respiratory epithelium. Eur Respir J 18(6):965-970. https:// doi.org/10.1183/09031936.01.00093001

20. Jiang RS, Liang KL, Hsin CH, Su MC (2016) The impact of chronic rhinosinusitis on sleep-disordered breathing. Rhinology 54(1): 75-79. https://doi.org/10.4193/Rhin15.204

21. Alt JA, Smith TL (2013) Chronic rhinosinusitis and sleep: a contemporary review. Int Forum Allergy Rhinol 3(11):941-949. https://doi.org/10.1002/alr.21217

22. Carpagnano GE, Spanevello A, Sabato R, Depalo A, Palladino GP, Bergantino L, Foschino Barbaro MP (2010) Systemic and airway inflammation in sleep apnea and obesity: the role of ICAM-1 and IL-8. Transl Res 155(1):35-43. https://doi.org/10.1016/j.trsl.2009. 09.004

23. Vgontzas AN, Papanicolaou DA, Bixler EO, Kales A, Tyson K, Chrousos GP (1997) Elevation of plasma cytokines in disorders of excessive daytime sleepiness: role of sleep disturbance and obesity. J Clin Endocrinol Metab 82(5):1313-1316. https://doi.org/10.1210/ jcem.82.5.3950

24. Alberti A, Sarchielli P, Gallinella E, Floridi A, Floridi A, Mazzotta G, Gallai V (2003) Plasma cytokine levels in patients with obstructive sleep apnea syndrome: a preliminary study. J Sleep Res 12(4): 305-311. https://doi.org/10.1111/j.1365-2869.2003.00361.x

25. Ciftci TU, Kokturk O, Bukan N, Bilgihan A (2004) The relationship between serum cytokine levels with obesity and obstructive sleep apnea syndrome. Cytokine 28(2):87-91. https://doi.org/10. 1016/j.cyto.2004.07.003

26. Shamsuzzaman AS, Winnicki M, Lanfranchi P, Wolk R, Kara T, Accurso V, Somers VK (2002) Elevated C-reactive protein in patients with obstructive sleep apnea. Circulation 105(21):24622464. https://doi.org/10.1161/01.cir.0000018948.95175.03

27. Ng KM, Lau CF, Fung ML (2010) Melatonin reduces hippocampal beta-amyloid generation in rats exposed to chronic intermittent hypoxia. Brain Res 1354:163-171. https://doi.org/10.1016/j.brainres. 2010.07.044

28. Shiota S, Takekawa H, Matsumoto SE, Takeda K, Nurwidya F, Yoshioka Y, Takahashi F, Hattori N, Tabira T, Mochizuki H, Takahashi K (2013) Chronic intermittent hypoxia/reoxygenation facilitate amyloid-beta generation in mice. J Alzheimer's Dis 37(2):325-333. https://doi.org/10.3233/JAD-130419

Publisher's note Springer Nature remains neutral with regard to jurisdictional claims in published maps and institutional affiliations. 